\title{
The Secure IEEE 11073:20601 Protocol and Agent Structure for Protecting PHD
}

\author{
Doyoung Chung ${ }^{1 *}$, Gaeil An ${ }^{2}$, Sokjoon Lee ${ }^{3}$, and Byungho Chung ${ }^{4}$ \\ ${ }^{* 1,2,3,4}$ Electronics and Telecommunications Research Institute, \\ 218 Gajeong-ro, Yuseong-gu, Daejeon, 34129, KOREA \\ 1*thisisdoyoung@etri.re.kr, ${ }^{2}$ fogone@etri.re.kr, ${ }^{3}$ junny@etri.re.kr, ${ }^{4}$ cbh@etri.re.kr
}

\begin{abstract}
In this paper, we suggest improvements of security in IEEE 11073:20601 protocol and the structure of its agent for protecting user's healthcare information safely. Current IEEE 11073:20601 standard does not provide any method to ensure security of data exchange, and it assumes that data exchange is secured by other means. The suggested improvements include mechanism to enhance security in exchanging and storing data in PHD. While providing those advantages, it also satisfies availability by effective encoding rules and data protecting algorithm.
\end{abstract}

Keywords: IEEE 11073, IEEE 11073:20601, PHD, Agent, Security

\section{Introduction}

Health concerns have been increasing in these days and willingness to use healthcare device has been increasing accordingly. Wearable health devices, such as a smart band, smart weight scale, are actualized as ICT technology has been developed.

For interoperability between healthcare devices, some organizations, such as Continua, IHE, IEEE, etc., are establishing protocols, frameworks, and standards. Healthcare services usually treat sensitive personal information, such as biometric and financial information, and some medical histories. For these reason, healthcare service is required to have a high level of security and this requirement also applies to Personal Healthcare Device(PHD)s.

IEEE established standards for PHD, which is called "IEEE 11073 [1]." IEEE 11073 standards enable communication between medical devices and external computer systems. IEEE 11073 is composed of IEEE 11073:20601 and IEEE 11073:104zz. IEEE 11073:20601 align with, and draw upon, the existing clinically focuses standards to provide easy management of data from either a clinical or personal health device. And IEEE 11073:104zz standards define specific device specializations.

The system model of IEEE 11073:20601 consists of agent and manager. In this paper, we expand IEEE 11073:20601 to improve security, and suggest the structure of IEEE 11073:20601 agent to protect users' more securely.

\section{Related Works}

IEEE 11073:20601 standard depicts security of this standard as depends on other means, for example, a secure transport channel. Such depiction has drawbacks, for example, the medical is leaked by the vulnerability of transport layer, and inefficient power and computing resource management by seperation between (security) transport layer and IEEE 11073:20601.

To overcome those drawbacks, several researches were conducted. Egner et al. suggest mechanism [2] for mutual authentication between IEEE 11073:20601 agent and manager. However, this mechanism does not provide protection mechanism for data exchange. A preliminary version of this paper appeared in ITCS 2016, July 5-8, Bali, Indonesia.

This version includes a thoughtful expansion of IEEE 11073:20601 for improving security. 
Rubio et al. suggest mechanism [3] for mutual authentication between IEEE 11073:20601 agent and manager and protection mechanism for data exchange. They suggest to provide security for the whole system as making it to interwork with Hospital Information System(HIS), and classify healthcare services by related components of the system depending on a certain standard such as (data) significance. Based on the classification, they propose security requirements for each classification and methods to fulfill each security requirement. However, their suggestion is based on the assumption that Public Key Certification is available on PHD.

By the researchs [4], [5], [6] which measure power consumption and time spending on Symmetric key encryption and Assymetric key encryption algorithm on the MSP430 platform, it requires about $4.91 \mathrm{~s}$ to using RSA-354 algorithm. Thus, the asymmetric key encryption algorithm, which is more securer than RSA-1024, is not available on PHD, which is usually using MSP430 platform.

On the other hand, symmetric key encryption algorithm, for example, AES-256 only consumes less than 1s to encrypt one block of plaintext. The secure hash algorithm also consume 35ms(SHA1) and 71ms(HMAC-SHA1) [7]. Therefore, we suggest our mechanism based on symmetric key encryption and secure hash algorithm.

\section{Secure IEEE 11073:20601 protocol}

The secure protocol which improve IEEE 11073:20601 is designed on the assumption that the agent and manager share Pre-shared Key(PSK) in advance. It can be achieved by the manufacturers or the users. The secure protocol is composed of Key agreement part based on IEEE 11073:20601 association and Data exchange protection part based on IEEE 11073:20601 operating state.

\subsection{Authentication and Key Exchangement}

In association stage, agent and manager generate session key based on the PSK. By modifying IEEE 11073:20601 to perform AARQ-AARE exchangement two times, 4-way handshake is performed betweeon agent and manager. By using Option-list field in AARQ, AARE in IEEE 11073:20601, we can transfer information related with key agreement 4-times. To insert those information in Option-list field, we defined those information as attribute as IEEE 11073:20601. In Table 1, we denotes each attribute by its name, id, type and qualifiers which required by IEEE 11073:20601

Table 1. Attributes for Authentication and Key Exchangement

\begin{tabular}{|l|l|l|l|}
\hline $\begin{array}{l}\text { Attribute } \\
\text { Name }\end{array}$ & Attribute ID & Attribute Type & Qualifiers \\
\hline $\begin{array}{l}\text { PSK } \\
\text { Identity }\end{array}$ & MDC_ATTR_SEC_PSK_IDENTITY & $\begin{array}{l}\text { OCTET } \\
\text { STRING }\end{array}$ & $\begin{array}{l}\text { Conditional } \\
\text { Dynamic }\end{array}$ \\
\hline Version & MDC_ATTR_SEC_PROTOCOL_VERSION & BITS-32 & $\begin{array}{l}\text { Mandatory } \\
\text { Static }\end{array}$ \\
\hline $\begin{array}{l}\text { Session } \\
\text { ID }\end{array}$ & MDC_ATTR_SEC_SESSION_ID & $\begin{array}{l}\text { OCTET } \\
\text { STRING(SIZE(32)) }\end{array}$ & $\begin{array}{l}\text { Conditional } \\
\text { Dynamic }\end{array}$ \\
\hline $\begin{array}{l}\text { Cipher } \\
\text { Suite }\end{array}$ & MDC_ATTR_SEC_CIPHER_SUITE & INT-U16 & $\begin{array}{l}\text { Mandatory } \\
\text { Dynamic }\end{array}$ \\
\hline Cipher & MDC_ATTR_SEC_CIPHER_SUITES & SEQUENCE OF & Mandatory \\
\hline
\end{tabular}




\begin{tabular}{|l|l|l|l|}
\hline Suites & & CipherSuite & Dynamic \\
\hline Finished & MDC_ATTR_SEC_FINISHED & $\begin{array}{l}\text { OCTET } \\
\text { STRING(SIZE(12)) }\end{array}$ & $\begin{array}{l}\text { Mandatory } \\
\text { Dynamic }\end{array}$ \\
\hline Random & MDC_ATTR_SEC_RANDOM & $\begin{array}{l}\text { OCTET } \\
\text { STRING(SIZE(32)) }\end{array}$ & $\begin{array}{l}\text { Mandatory } \\
\text { Dynamic }\end{array}$ \\
\hline
\end{tabular}

According to IEEE 11073:20601 nomenclature and IEEE 11073:10101, the term codes from 0xF000 - 0xFFFF are reserved for private or vendor-specified nomenclature codes. Our suggestion is not accepted to IEEE 11073:20601 standard yet, thus we use private nomenclature codes for our implementation. Correspoding code for each Attribute is depicted in Table 2.

Table 2. Attribute Codes for Authentication and Key Exchangement

\begin{tabular}{|l|l|}
\hline Attribute ID & Code \\
\hline MDC_ATTR_SEC_PSK_IDENTITY & 0xF0 0x01 \\
\hline MDC_ATTR_SEC_PROTOCOL_VERSION & 0xF0 0x02 \\
\hline MDC_ATTR_SEC_SESSION_ID & 0xF0 0x03 \\
\hline MDC_ATTR_SEC_CIPHER_SUITE & 0xF0 0x04 \\
\hline MDC_ATTR_SEC_CIPHER_SUITES & 0xF0 0x05 \\
\hline MDC_ATTR_SEC_FINISHED & 0xF0 0x06 \\
\hline MDC_ATTR_SEC_RANDOM & 0xF0 0x07 \\
\hline
\end{tabular}

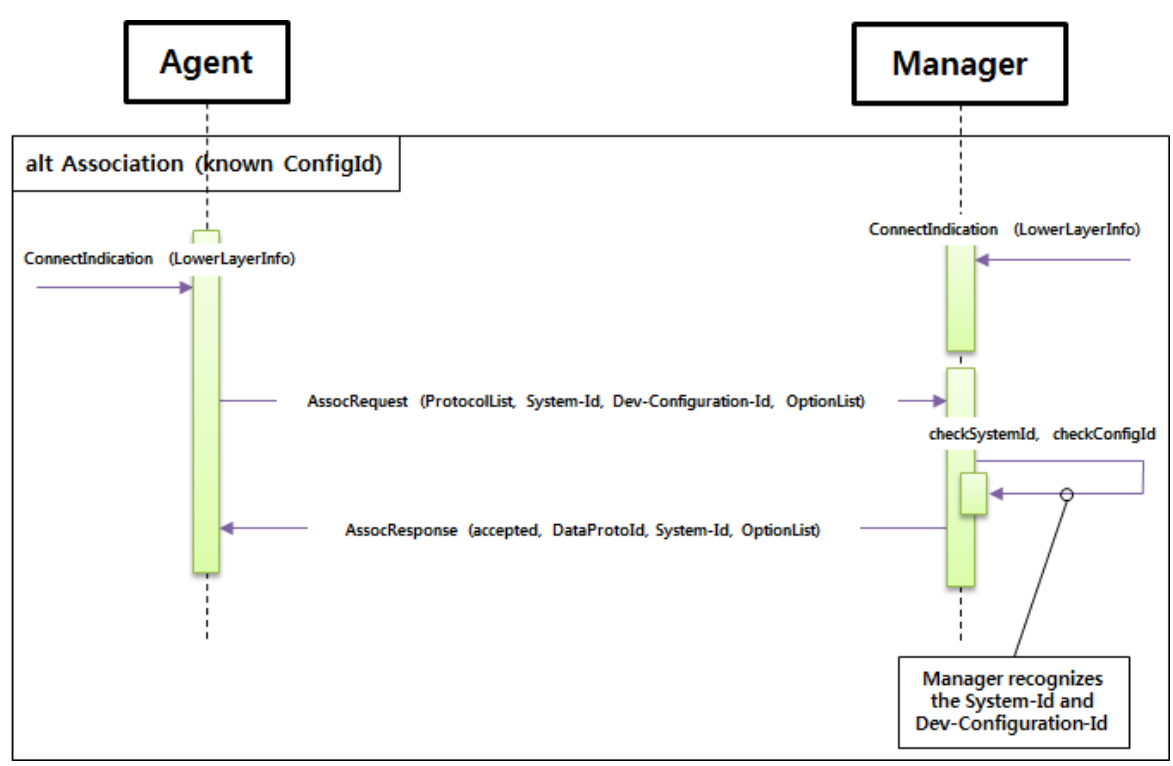

Figure 1. The Procedure of Legacy IEEE 11073:20601 Association

Originally, the association procedure of IEEE 11073:20601 is 2-way handshake of AARQ and AARE. Figure 1 depicts the association procedure of IEEE 11073:20601. We modify the association procedure to send AARQ and AARE two times. Moreover we 
expand AARQ and AARE by using its Option-list field. Option-list field is used to communicate additional attributes between agent and manager. Thus insertion defined attributes in Option-list field is coincide with the purpose of Option-List. The expansion of assocation procedure for our protocol is depicted in Figure 2. By this expansion we retain 4-way handshakes and the space for containing additional information which required to authentication and key exchangement.

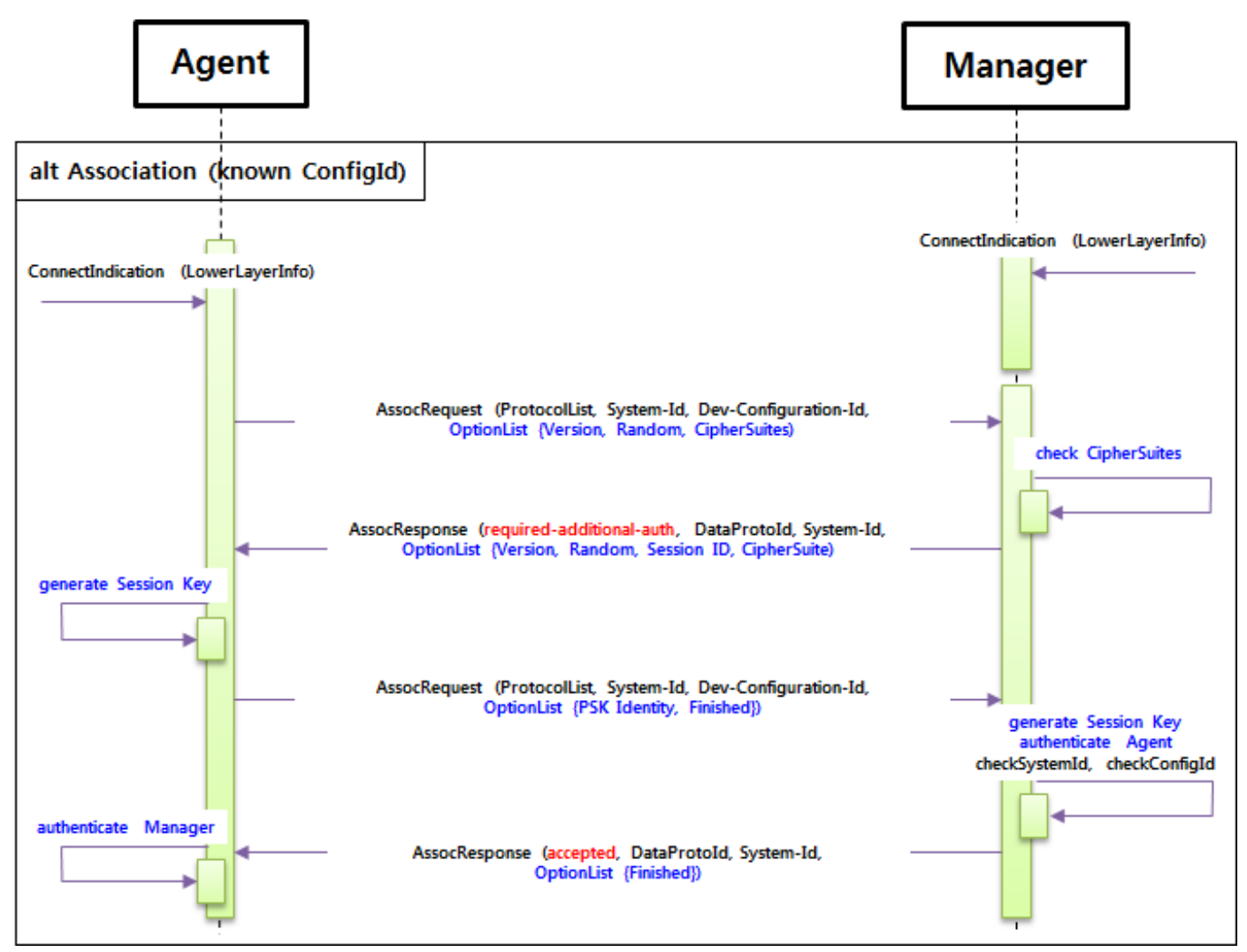

Figure 2. The Procedure of Secure IEEE 11073:20601 Association

Thus it can be merged with existing key agreement mechanisms based on 4-way handshake, for example, handshake protocol in TLS [8], [9], [10]. While associating, the agent and manager perform agreement for two keys. One is session key, which for protecting data exchangement. The other is PM-store key, which for protecting data which store on PHD locally.

The PM-store key is used for encrypt medical data which located on PM-store object. Generally, the medical data which located on PM-store is stored on PHD locally. Thus it is used for protecting medical data from data theft by local access. It is discarded from PHD, when the connection with manager is disconnected. In other words, data thief takes the PHD from original place then the PM-object key is discared. For example, the PMstore key is discared when the PHD is moved more than few tens of meter for Bluetooth connection.

Our protocol supports two types of handshake for authentication and key exchagement method. Full handshake is default. It is depicted in Figure 2. Abbreviated handshake can reduce the procedure for authentication and key exchangement to 3-way handshakes. This handshake is used for restore session which generated by Full handshake. The agent and manager store session information for restoration, and use it when they have to reconnect. The information for restoring session is as follow, Session ID, Session Key, and Cipher 
Suite. The Session Key is composed of Encryption Key and MAC Key which used for message protection. And it generated by Master Secret.

The differnece between Full and Abbreviated Handshake is depicted in Figure 3. The left sequence diagram represents the attributes which contained in Option-list for Full handshake, and the right one represents those for Abbreviated Handshake. In stage 3 of Full handshake, agent and manager generate master secret by using random numbers and PSK. On the other hand, in Abbreviated Handshake, the agent and manager already have master secret. Thus they can reduce authentication and key exchangement procedure to 3way handshake. More specific explanation of Abbreviated Handshake is existed on TLS standards.
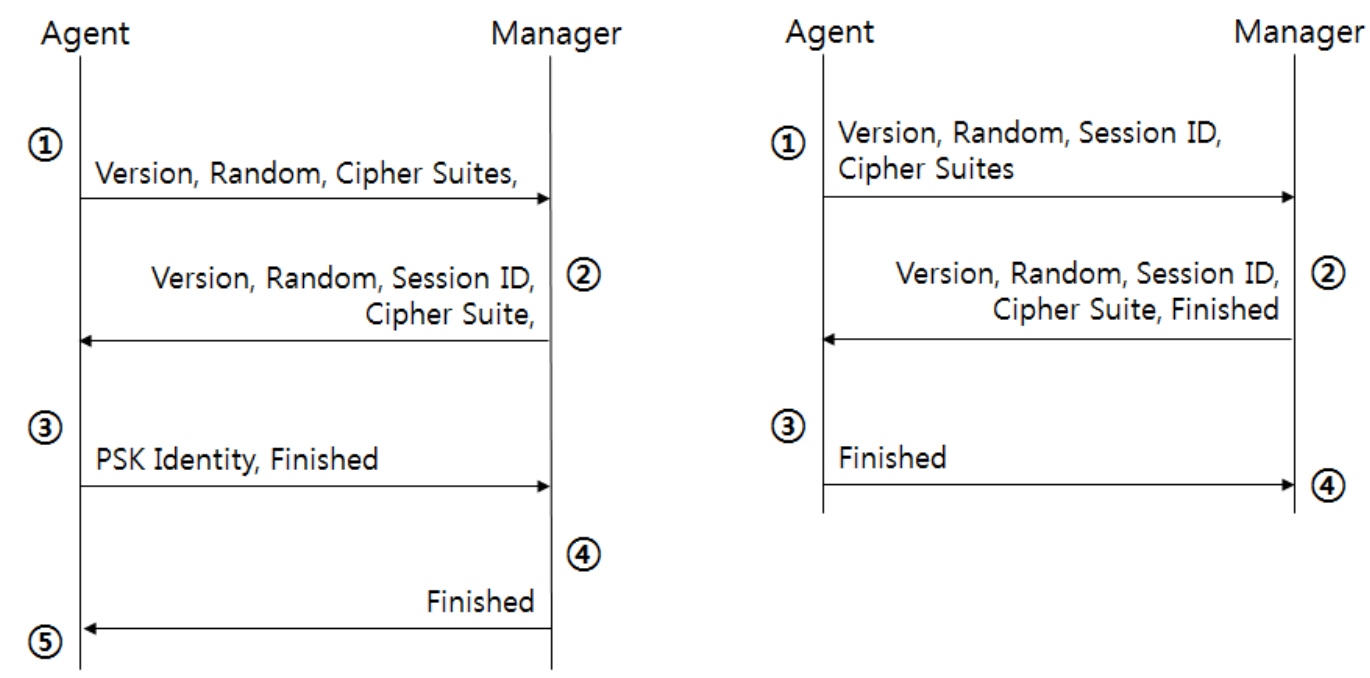

Figure 3. Comparison between Full and Abbreviated Handshake

As mentioned above, security of IEEE 11073:20601 depends on other means, for example, a secure transport channel. Wide spreaded wireless transport channel, such as, Bluetooth and WiFi also requires 4-way handshake for its secure connection. Thus legacy IEEE 11073:20601 requires 6-way handshake for its secure connection totally. 4-way handshake for secure transport channel, and 2-way handshake for IEEE 11073:20601 itself. We embedded security mechanisms by expanding association procedure in IEEE 11073:20601, thus it requires only 4-way handshake for Full handshake, and 3-way handshake for Abbreviated Handshake. Moreover the implementation of IEEE 11073:20601 Agent send medical data occasionally, thus it does not maintain its connection with Manager. By this reason, suggested abbreviated handshake is used often and it requires only half-of-handshake than legacy IEEE 11073:20601.

\subsection{Message Protection}

For exchanging data securely, the secure protocol follows MAC-then-Encrypted security mechanism [11]. We use CBC-mode block cipher, such as AES-128-CBC, AES256-CBC message encryption. This meachanism is despicted in Figure 4. It transforms PRST message in IEEE 11073:20601 to protected form which named Secure PRST message. It is applied on operating state in IEEE 11073:20601. 


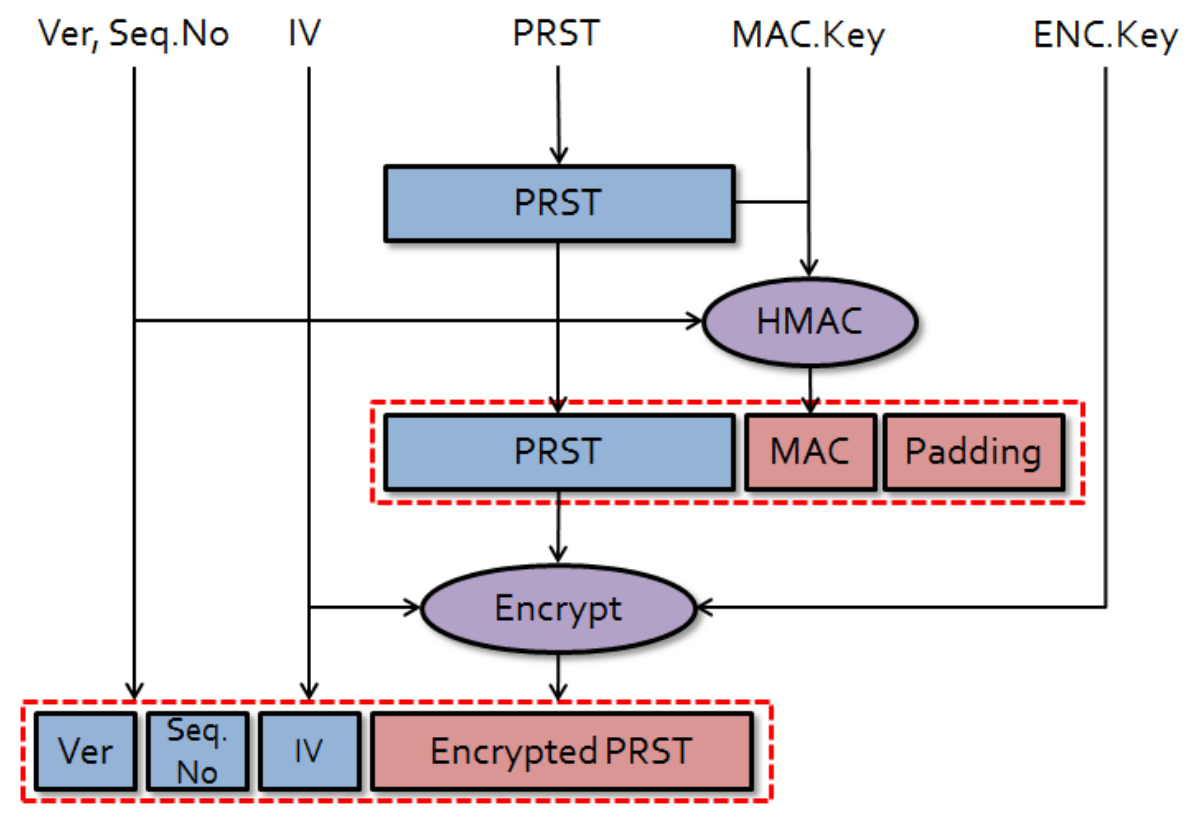

\section{Figure 4. The Procedure of Transformation PRST to Secure PRST}

We define Secure PRST folowing MDER encoding which must be supported by the agent. The definition of Secure PRST by ASN.1 notation is as follow.

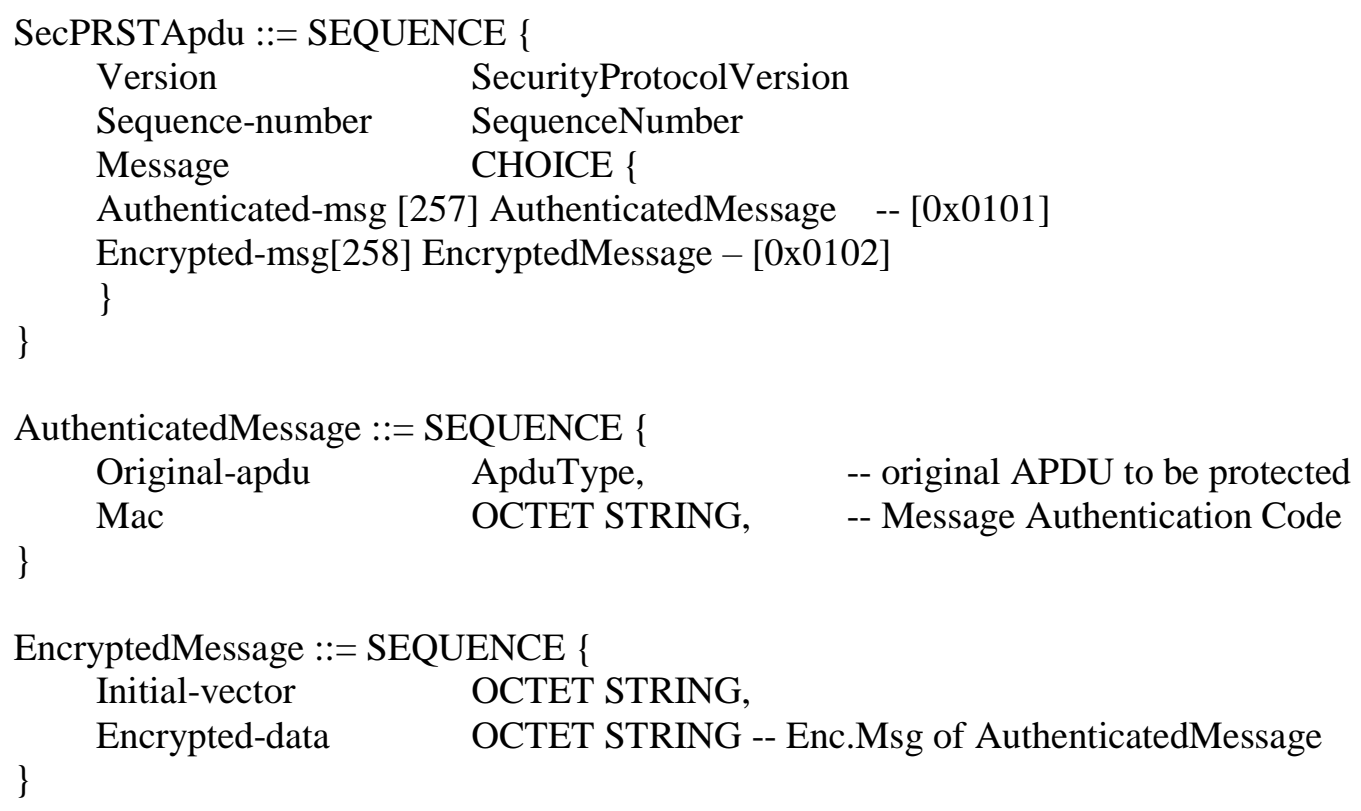


We propose AuthenticatedMessage and EncryptedMessage for Secure PRST. AuthenticatedMessage supports only message authentication algorithm. It cannot suggest confidentiality. But we assume that under the emergency situation, the agent has to connect to the manager even though the manager does not support any encryption algorithm.

On the other hand, EncryptedMessage support confidentiality, integrity, and availability. It also has resistance against reply attack by including sequenbe number in HMAC and EncryptedMessage.

\section{Structure of Secure IEEE 11073:20601 Agent [12]}

The structure of Secure IEEE 11073:20601 is consist of legacy IEEE 11073:20601 agent, Secure Device Specification Block(SDSB), and Secure Message Exchange Block(SMEB).

SDSB, and SMEB hooking the message between Network Interface and Application Layer which is legacy IEEE 11073:20601 agent. This structure seperates legacy IEEE 11073:20601 agent and suggested secure mechanism by layering. Thus it eases to apply secure mechanism on legacy PHD. We apply our proposed secure mechanism on legacy IEEE 11073:20601 agent emulator and prove its easiness for apply legacy PHD.

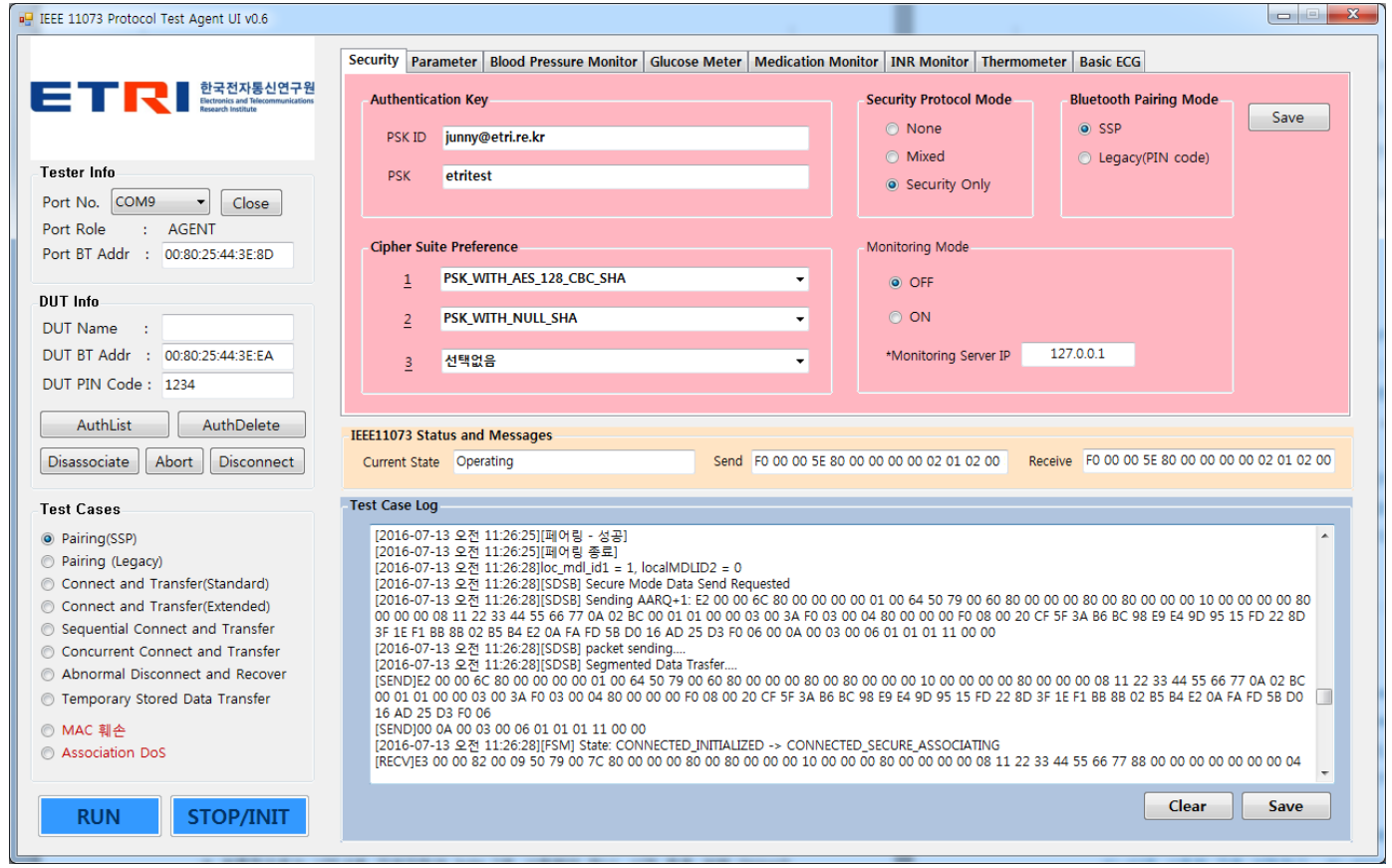

Figure 5. The Implementation of Secure IEEE 11073:20601 Agent

Figure 6 depicts the structure of SDSB. SDSB manages association and configuring state in Secure IEEE 11073:20601. Moreover it supports the protection mechanism which related with each device specification. For example, it supports the protection mechanism for PM-store Object. SDSB hooks the association and configuring state in IEEE 11073:20601, and performs 4-way handshake by using modified AARQ-AARE exchangement two times. 


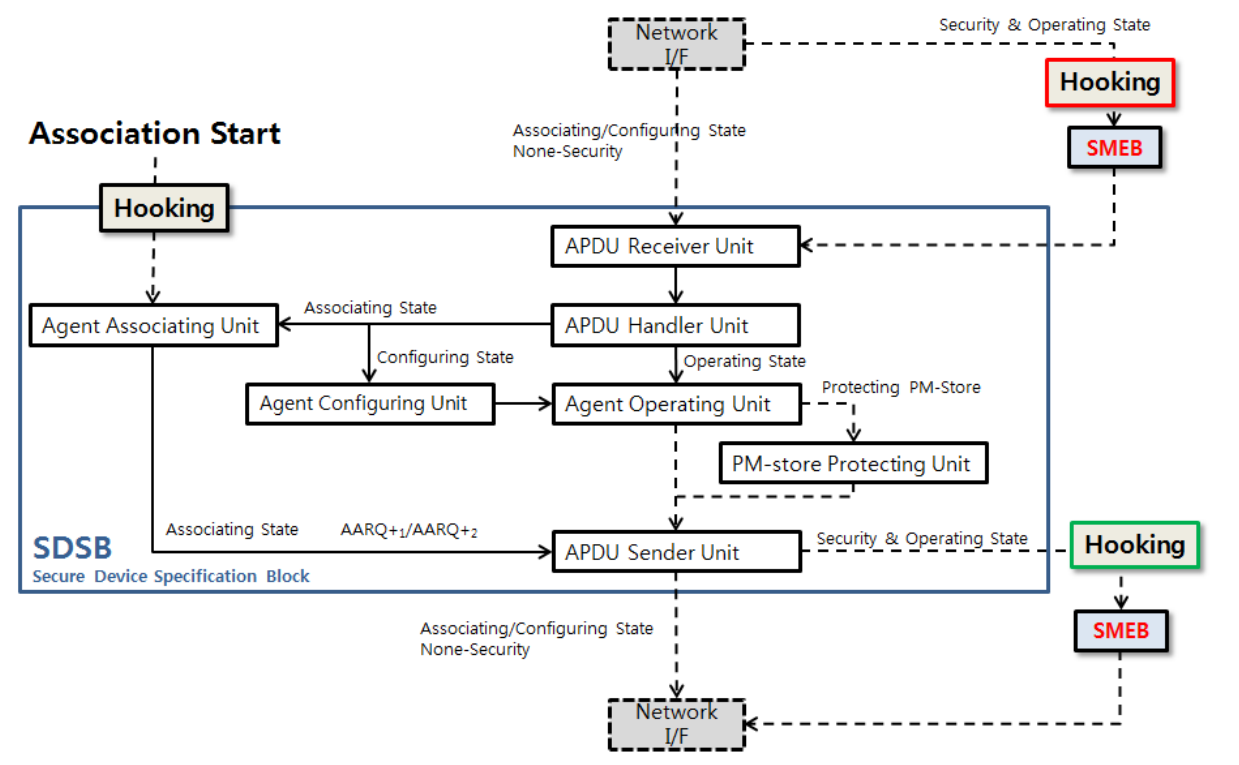

Figure 6. The Structure of Secure Device Specification Block (SDSB)

In operating state, Secure IEEE 11073:20601 Agent provides two secure mechanisms. One is protection of PM-Store Object, and the other is protection of message exchange. The protection of PM-Store Object is achieved by encrypt medical data in PM-Store by PM-Store key, and store it on local storage. The protection of message exchange is performed by SMEB.

Figure 7 depicts the structure of SMEB. SDSB and SMEB have to interwork, and the interworking point is depicted in both Figure 6 and Figure 7. SMEB hooks PRST message generated by legacy IEEE 11073:20601 agent. Then it transforms the PRST message to Secure PRST message as depicted in Figure 4. SMEB is consists of Secure PRST unit, HMAC unit, Symmetric Encryption/Decryption Unit, and Validation Unit.

The role of HMAC unit and Symmetric Encryption/Decryption unit is obvious. The Secure PRST unit receives HMAC, encrypted message which generated by above units. Then it assembles Secure PRST message with protocol version, sequence number of messages, and initial vector for CBC-mode block cipher. The initial vector should be generated randomly for every message. When the Secure PRST message is recived, Secure PRST unit parses it and seperates each element.

The Validation unit performs comprehensive validation on each Secure PRST message. It validates each Secure PRST message with HMAC, and checks whether decyprted message is PRST or not. Currently, the main role of Validation unit is detection of replay attack using sequence number in Secure PRST message. However we expect that the role of Validation unit is expanded in further works, and the availability of detecting attack is improved. 


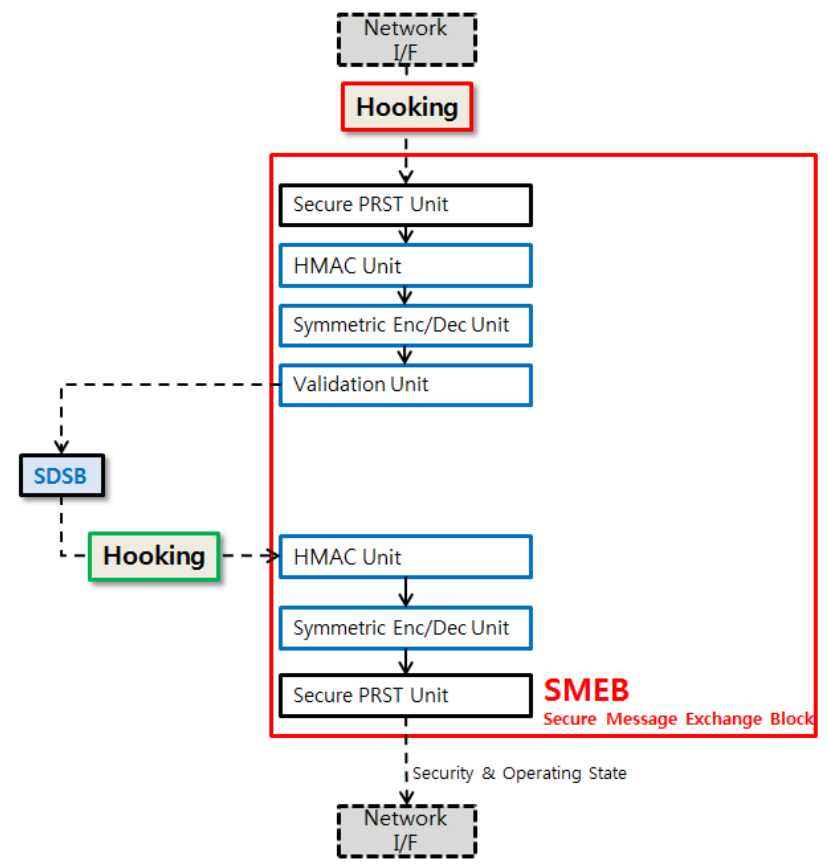

Figure 7. The Structure of Secure Message Exchange Block (SMEB)

The Validation unit performs comprehensive validation on each Secure PRST message. It validates each Secure PRST message with HMAC, and checks whether decyprted message is PRST or not. Currently, the main role of Validation unit is detection of replay attack using sequence number in Secure PRST message. However we expect that the role of Validation unit is expanded in further works, and the availability of detecting attack is improved.

\section{Analysis of Proposed Scheme}

We analysis our proposed scheme has resistance against well-known attack in this chapter. Our proposed scheme satisfies confidentiality, integrity, and availability. It is based on PSK, thus it also satisfies mutual authentication. By those characteristics, our proposed scheme is appropriate for keep user's privacy and safety.

\subsection{Eavesdropping Attack}

A different session key is generated for each session, and the session key is not possible to suspect from the knowledge of past session keys. In our scheme, the session key is calculated by agent random, manager random, and PSK. Until PSK is not exposed, then the adversary cannot generate session key. Our proposed scheme, the message which contains medical data is protected by well-proved encryption algorithms, such as AES128-CBC, AES-256-CBC, etc.

\subsection{MITM attack}

Our proposed scheme satisfies end-to-end security, because only validated agent and manager know PSK. The agent and manager authenticate each other based on PSK. Even the agent random and manager random are exposed during association procedure, but the session key is generated by using PSK also. Thus the adversary cannot mimic as validated agent or manager. 


\subsection{Replay Attack}

Our proposed scheme includes varying part for every message. The sequence number is included in Secure PRST. It used for generating HMAC for each secure PRST. Moreover it appended to legacy IEEE 11073:20601 PRST and encrypted together. Thus the adversary cannot generate Secure PRST by using preferred sequence number. The adversary also cannot reuse Secure PRST, because the sequence number has to be increased for every message.

\section{Conclusion}

In this paper, we present the expansion of IEEE 11073:20601 for improving security. We also suggest and implement the structure of IEEE 11073:20601 agent for protecting PHD. Unfortunately most of PHD is based on MSP430 platform, and this platform is hard to support Asymmetric Key based crypto primitives. Thus previous works on protecting IEEE 11073:20601 agent, manager, or connection have difficult to apply on low power, low performance devices.

We suggest secure mechanisms for protecting medical data which stored on PHD locally, and protecting data exchange between IEEE 11073:20601 agent and manager. Our mechanism is based on crypto primitives using symmetric key. Thus it satisfies availability especially, while satisfies other security requirements, such as, confidentiality and integrity. In chapter 4, we explain how our suggested mechanism provides resistance against security threats, such as Eavesdropping, MITM, Replay attacks. Moreover our mechanism provides expandability to support end-to-end security.

The advantage of our approach is providing protection mechanism for local stored data, while protecting data exchanging. Moreover it is appropriated to apply on actual PHD, which has low power and low performance. For further works, we are planning to improve secure mechanisms for local stored data, for example, restoring PM-store Key by resuming previous Session.

\section{Acknowledgments}

This work was supported by Institute for Information \& communications Technology Promotion(IITP) grant funded by the Korea government(MSIP) (No.B0713-15-0007, Development of International Standards Smart Medical Security Platform focused on the Field Considering Life Cycle of Medical Information)

\section{References}

[1] IEEE, "IEEE Std 11073-20601TM-2014, Health informatics - Personal health device communication Part 20601: Application profile - Optimized Exchange Protocol", (2014).

[2] Alexandru Egner, Alexandru Soceanu and Florica Moldoveanu, "Managing Secure Authenticatio for Standard Mobile Medical Networks", Proceedings of the 7th IEEE Symposium on Computers and Communications, Cappadocia, Turkey, (2012) July 1-4.

[3] Oscar J. Rubio, Jesus D. Trigo, Alvaro Alesanco, Luis Serrano and Jose Garcia, “Analysis of ISO/IEEE 11073 built-in security and its potential IHE-based extensibility”, Journal of Biomedical Informatics., vol. 60, (2016), pp. 270-285.

[4] Benjamin Buhrow, Paul Riemer, Mike Shea and Barry Gilbert, Erik Daniel, "Block Cipher Speed and Energy Efficiency Records on the MSP 430: System Design Trade-Offs for 16-Bit Embedded Applications", Progress in Cryptology - LATINCRYPT 2014, (2015), pp. 104-123.

[5] Ondrej Hyncica, Pavel Kucera, Petr Honzik and Petr Fiedler, "Performance Evaluation of Symmetric Cryptography in Embedded Systems", Proceedings of the 6th IEEE International Conference on Intelligent Data Acquisition and Advanced Computing Systems: Technology and Applications, Prague, Czech Republic, (2011) September 15-17.

[6] Gustavo S. Quirino, Edward David Moreno and Leila B. C. Matos, "Performance Evaluation of Asymmetric Encryption Algorithms in embedded platforms used in WSN", Proceedings of the 12th International Conference on Security and Management, Las Vegas, USA, (2013) July 22-25. 
[7] HangRok Lee, YongJe Choi and HoWon Kim, "Implementation of TinyHash based on Hash Algorithm for Sensor Network", International Journal of Electrical, Computer, Energetic, Electronic and Communication Engineering., vol. 1, no. 2, (2007),pp. 1564-1568.

[8] T. Dierks and C. Allen, "The TLS Protocol Version 1.0", IETF Standard, RFC 2246, (1999).

[9] T. Dierks and E. Rescorla, "The Transport Layer Security (TLS) Protocol Version 1.1”, IETF Standard, RFC 4346, (2006).

[10] T. Dierks and E. Rescorla, "The Transport Layer Security (TLS) Protocol Version 1.2", IETF Standard, RFC 5246, (2008).

[11] P. Eronen and H. Tschofenig, "Pre-shared Key Ciphersuites for Transport Layer Security (TLS)", IETF Standard, RFC 4279, (2005).

[12] Doyoung Chung, Gaeil An, Sokjoon Lee and Byungho Chung, "The study on structure of IEEE 11073:20601 Agent for protecting PHD", Proceedings of the 5th International Conference on Information Technology and Computer Science, Bali, Indonesia, (2016) July 5-8.

\section{Authors}

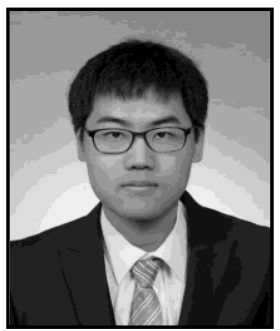

Doyoung Chung, he received B.E. and M.S. degress in computer science from KAIST, Republic of Korea. Since 2012, he has been a researcher in Electronics and Telecommunications Research Institute. His interests include Cryptography, Wireless Protocol Security, Deep-Learning for Security and Privacy of Healthcare Information.

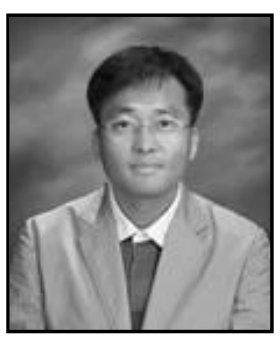

Gaeil An, he received M.S. and Ph.D. degree in computer Engineering from the Chung-Nam National University, in 1995, and in 2001, respectively. He is a Senior Engineer at Information Security Research Division, ETRI, and has worked in ETRI since 2001. His interest area includes Network Security, Healthcare Security, and Network Simulation.

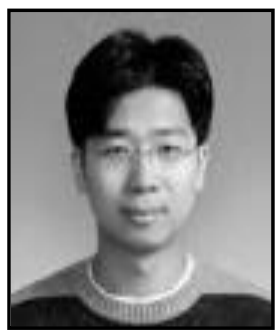

Sokjoon Lee, he received his Bachelor's and Master's Degree in Computer Engineering from Seoul National University, in 1998, and in 2000, respectively. He is working as a Principal Researcher in Electronics and Telecommunications Research Institute and also studying his Ph.D. degree of Computer Science in KAIST, Korea. His interests include Cryptography, Wireless Protocol Security and Privacy of Medical/Healthcare Information.

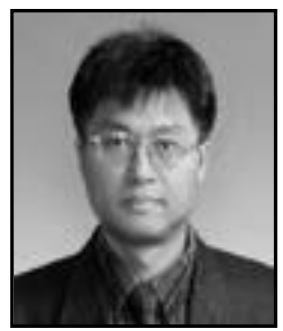

Byungho Chung, he received M.S. and Ph.D. degree in computer Engineering from the Chung-Nam National University, in 2000, and in 2005, respectively. He is working as a Principla Researcher and Lab Leader in Electronics and Telecommunications Research Institute. His interest area includes Wireless Protocol Security, Security in maritime system, Smart car Security and Secure Medical/Healthcare system. 
International Journal of Security and Its Applications

Vol. 10, No. 12 (2016) 